\title{
Nanofluidic Cells With Controlled Path Length and Liquid Flow for Rapid, High- Resolution in situ Electron Microscopy.
}

C. Mueller ${ }^{1,2}$, M. Harb ${ }^{2}$, J.R. Dwyer ${ }^{2,3}$, R.J.D. Miller ${ }^{1,4}$

1 Departments of Chemistry and Physics, University of Toronto, Toronto, Canada

${ }^{2}$ Insight Nanofluidics Inc, Toronto, Canada

${ }^{3}$ Department of Chemistry, University of Rhode Island, Kingston, USA

${ }^{4}$ Max Planck Research Group for Structural Dynamics, Department of Physics, University of Hamburg, c/o DESY, Hamburg, Germany.

In situ imaging using (scanning) transmission electron microscopes has proven to be an extremely important and powerful cross-disciplinary scientific technique. In particular nanotechnology and materials sciences have special interest in assembly and disintegration processes, in growth and shapetuning of (nano)-particles, ${ }^{1,2}$ and, furthermore in mechanistic studies of chemical reactions underlying these processes. However, limitations for in liquid and in situ imaging utilizing electron microscopy arise from experimental conditions required to minimize disturbing electron scatter. Within current sample preparation methods, these limitations are difficult to achieve: ${ }^{3}$ Nanometer thin and vacuum compatible samples, which are additionally easy to use, reliable and provide relatively high-throughput flow are pending.

Here, we present a nanofluidic sample cell allowing for exquisite control over the liquid layer thickness, currently $>40$ nanometer, in order to preserve the highest possible spatial resolution for the in situ study of liquid/solutions using electron microscopy. Preparing such ultra thin liquid layers enables us to use a variety of electron microscopes with different lens configurations and electron energies for our imaging experiments. We provide liquid flow through our nanocell by applying differential pressure with feedback control external to the microscope column and therewith allowing for on-the-fly sample exchange within the imaging area (Figure 1).

In this contribution, we show the ability to clearly image gold nanoparticles as small as 5nm, and polymer-based nanoparticles as small as $36 \mathrm{~nm}$, over fluid layers as thin as $50 \mathrm{~nm}$. Thicker layers, up to $280 \mathrm{~nm}$, show clear degradation in spatial resolution but still deliver good quality imaging. We studied the imaging resolution dependent on the liquid layer thickness, obtaining a linear relation between the two (Figure 2).

Furthermore, we demonstrate unidirectional flow in the design concept using gold nanorods pumped directionally through the imaging area upon an applied external force, while tumbling in Brownian motion once the external pressure is released. Having shown the capabilities of our nanofluidic design, we further show preliminary results of in situ imaging, studying gold nanorods to illustrate applications in the study of materials and amyloid fibrils as example of biological applications. Taken together, these systems highlight the variety of applications our nanofluidic cell is able to address. This design is distinguished by straightforward, reliable operation in different, commonly used electron microscopes. In future experiments, we will expand the use of our unique ability to control the path thickness and flow directionality. The latter will be key to enable time resolved electron diffraction in the liquid phase, allowing studies of a wide range of chemical reaction mechanisms on an atomic or molecular level. 
A)

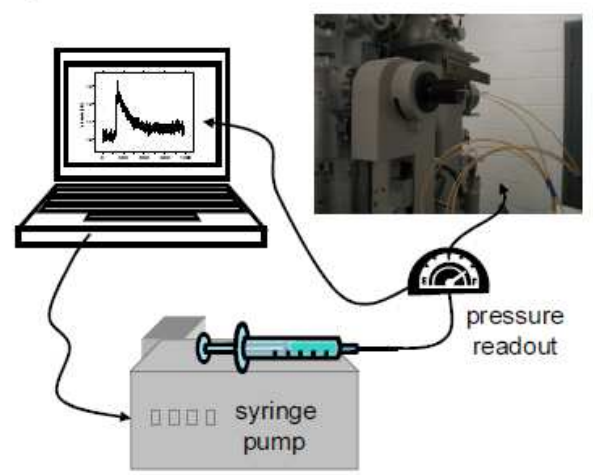

B)

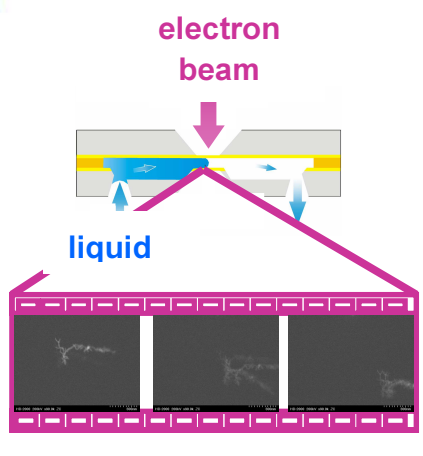

Figure 1. Experimental Setup. A) Schematic of the feedback loop to control the liquid flow conditions within the nanocell. B) Schematic of the nanofluidic cell showing the liquid flow path for the liquid specimen entering the nanocell and the resulting imaging sequence with flow condition.

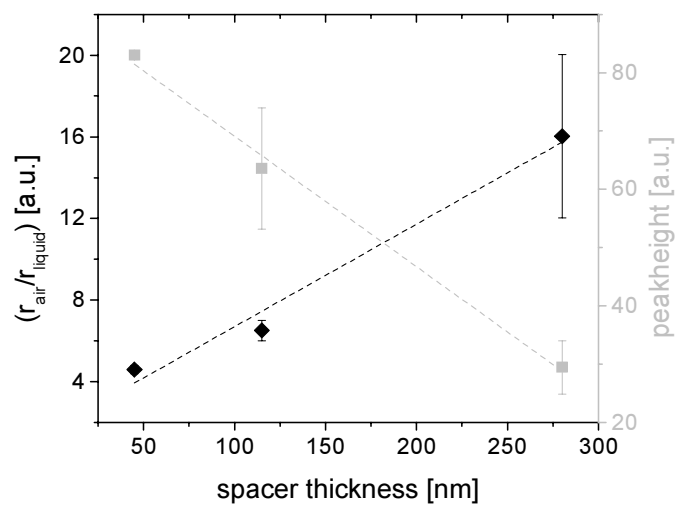

Figure 2. Relationship of resolution loss and liquid layer thickness. Linear dependence of the liquid layer thickness to the resolution loss ratio on left $y$-axis (black) and respective peak heights within the TEM images on the right y-axis (grey).

References:

[1] Yuk, J. M. et al. High-Resolution EM of Colloidal Nanocrystal Growth Using Graphene Liquid Cells. Science 336, 61-64 (2012).

[2] Grogan, J., Rotkina, L. \& Bau, H. In situ liquid-cell electron microscopy of colloid aggregation and growth dynamics. Physical Review E 83, 061405 (2011).

[3] deJonge, N. \& Ross, F. M. Electron microscopy of specimens in liquid. Nature Nanotechnolgy 6, 695-704 (2011).

[4] CM is supported through a Vanier Scholarship. The authors acknowledge financial support through NSERC and Insight Nanofluidics. 